\title{
Many-body effects on the electronic and optical properties of strained semiconducting carbon nanotubes
}

\author{
Catalin D. Spataru and François Léonard \\ Sandia National Laboratories, Livermore, California 94551
}

\begin{abstract}
We present many-body $a b$ initio calculations of the electronic and optical properties of semiconducting zigzag carbon nanotubes under uniaxial strain. The GW approach is utilized to obtain the quasiparticle bandgaps and is combined with the Bethe-Salpeter equation to obtain the optical absorption spectrum. We find that the dependence of the electronic bandgaps on strain is more complex than previously predicted based on tight-binding models or density-functional theory. In addition, we show that the exciton energy and exciton binding energy depend significantly on strain, with variations of tens of meVs per percent strain, but that despite these strong changes the absorbance is found to be nearly independent of strain. Our results provide new guidance for the understanding and design of optomechanical systems based on carbon nanotubes.
\end{abstract}

\section{INTRODUCTION}

The electronic and optical properties of nanomaterials such as carbon nanotubes (CNTs), graphene, and nanowires show unique behavior due to their reduced dimensions. For example, the electronic properties of CNTs depend strongly on their diameter [1], and many-body effects are known to significantly increase CNT bandgaps compared to density functional theory (DFT) 2]. In addition, CNT optical properties are dominated by excitons [2 7] due to a combination between weak electrostatic screening [1] and enhanced Coulomb effects in quasi-one-dimensional systems [2, 3].

While the electronic and optical properties of isolated CNTs are well understood, external factors such as the dielectric environment [5, 8], electrostatic doping [9, 10], and nanotube-nanotube interactions 11], have recently been shown to modify CNT electronic and optical properties. Another important external factor that impacts CNT properties is strain. Indeed, it was recognized early on [12, 13] that strain can significantly modify CNT electronic properties, and this has been exploited to realize new types of nanoelectromechanical devices 14]. However, to date theoretical studies of the impact of strain on CNT electronic properties have been mostly limited to tight-binding models and DFT; given the importance of many-body effects in unstrained CNTs, a question to address is the role of many-body effects in strained CNTs.

The role of many-body effects on the optical properties of strained CNTs has received even less attention. Experimental reports of strain modulation of CNT optical properties have recently emerged $15-17]$, and indicate that optical transition energies can shift by tens of meVs per percent strain. However, interpretation of these results has relied on non-interacting models developed for electronic transitions, which do not capture excitonic effects that dominate the optical response in CNTs. Progress in developing exciton-based models for the optical properties of strained CNTs has focused on approaches relying on the tight-binding or the $\mathbf{k} \cdot \mathbf{p}$ methods [18 21]. However, a full many-body $a b$ initio calculation of CNT optical properties under strain is still missing.

In this paper, we present such calculations by combining the GW approach with the Bethe-Salpeter (BSE) equation to study the electronic and optical properties of strained semiconducting CNTs. We find that the dependence of the electronic bandgaps on strain is more complex than previously predicted based on tight-binding models or density-functional theory. In addition, we show that the exciton energy and exciton binding energy depend significantly on strain, with variations of tens of meVs per percent strain. Furthermore, the absorbance is found to be nearly independent of strain as a consequence of the increase in transition dipole matrix elements with increasing strain.

This paper is organized as follows. After this Introduction, section II describes the methodology and results for the electronic properties of strained CNTs. Section III discusses the methodology and results for the optical spectra, exciton energies, and excition binding energies. A summary is presented in Section IV.

\section{IMPACT OF MANY-BODY EFFECTS ON ELECTRONIC PROPERTIES}

We perform our ab initio calculations on the semiconducting $(11,0)$ and $(17,0)$ CNTs for uniaxial strains from $0 \%$ to $5 \%$. We start by investigating the ground-state properties (e.g. relaxed atomic structure, electron density) within DFT. The DFT calculations are performed using the Quantum Espresso package 22] within the Local Density Approximation (LDA), using ab initio pseudopotentials in combination with a plane-wave basis set with a kinetic energy cutoff of 60 Ryd, in a supercell geometry with tube separation (center to center) of more than double the nanotube diameter. The atomic structure is relaxed until forces are smaller than $5 \mathrm{meV} / \AA$ for both the strained and unstrained cases. In the unstrained case the $(11,0)$ and $(17,0)$ CNTs have diameters of 8.6 and $13.2 \AA$ respectively. The strain is applied by stretching (w.r.t. to the unstrained case) the nanotube unit cell lattice vector along the tube axis followed by 
atomic relaxation. This leads to a decrease in nanotube diameter and a Poisson ratio $\nu \approx 0.15$.
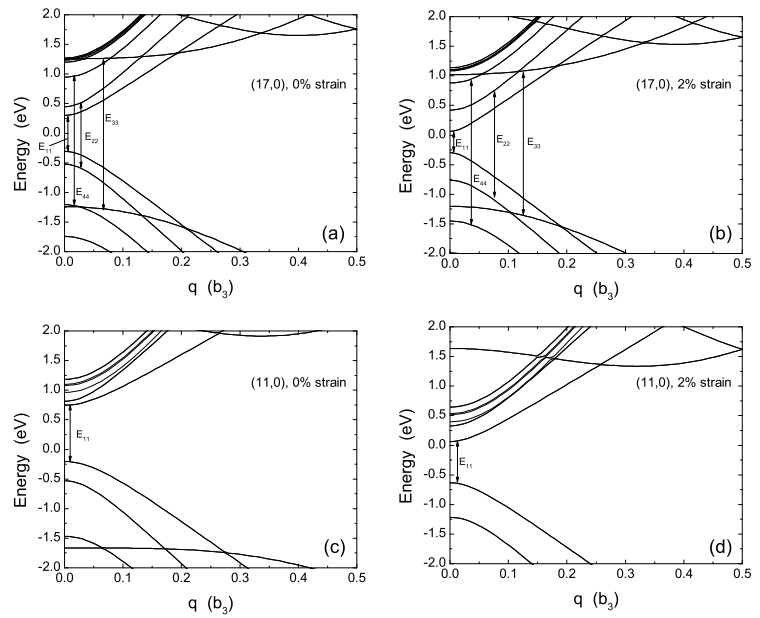

FIG. 1: DFT (LDA) bandstructure of the $(17,0)$ and $(11,0)$ CNTs at $0 \%$ and $2 \%$ strain. The electronic bandgaps and optical transitions studied in this paper are indicated with arrows. $b_{3}$ is the length of the Brillouin zone.

Figure 1 shows the DFT bandstructures at $0 \%$ and $2 \%$ strain, with the electronic bandgaps and optical transitions studied in this paper labelled in the figure. We note that for the unstrained $(17,0) \mathrm{CNT}$ the $E_{33}$ transition has higher energy than the $E_{44}$ transition due to trigonal warping [23]. Within LDA (Table 1), the $(17,0)$ fundamental bandgap is found to decrease with strain with a change $\Delta E_{g}^{11}$ of $-125 \mathrm{meV} / \%$, in agreement with previous DFT calculations 24, 25]. The higher energy gaps show changes $\Delta E_{g}^{22}=+108 \mathrm{meV} / \%, \Delta E_{g}^{33}=-136 \mathrm{meV} / \%$, and $\Delta E_{g}^{44}=+83 \mathrm{meV} / \%$. These values can be compared with those obtained from the simple tight-binding (TB) expression for small strain[26] applied to zigzag CNTs

$$
\Delta E_{g}^{k k}=(-1)^{k} 3 \gamma(1+\nu) \sigma,
$$

where $\gamma$ is the tight-binding overlap integral and $\sigma$ is the strain. Reasonable agreement with the LDA values can be obtained if one uses $\gamma=3.3 \mathrm{eV}$ and $\nu=0.15$, giving $\Delta E_{g} / \sigma= \pm 114 \mathrm{meV} / \%$.

While LDA and TB calculations agree to a large extent, an open question is whether many-body effects can change the above picture. To address this question, we performed quasiparticle calculations using the many-body GW approach 27]. The electron self-energy $\Sigma=i G W$ is obtained within the $G_{0} W_{0}$ approximation, i.e. using the LDA eigenvalues and wavefunctions to construct the 1-particle Green's function $G$. The screened Coulomb interaction $W$ is evaluated within the Random Phase Approximation and extended at non-zero frequencies using the Plasmon-Pole approximation [27]. We consider empty states up to an energy cutoff of $\sim 60 \mathrm{eV}$, and use the 'static-remainder' technique 28] to ensure convergence with respect to the number of empty states.

\begin{tabular}{|c|c|c|c|c|c|}
\hline & Strain (\%) & $\mathrm{E}_{11}(\mathrm{eV})$ & $\mathrm{E}_{22}(\mathrm{eV})$ & $\mathrm{E}_{33}(\mathrm{eV})$ & $\mathrm{E}_{44}(\mathrm{eV})$ \\
\hline \multirow[t]{2}{*}{ LDA } & 0 & 0.606 & 0.968 & 2.495 & 2.153 \\
\hline & 2 & 0.356 & 1.184 & 2.223 & 2.329 \\
\hline \multirow[t]{2}{*}{ GW } & 0 & 1.291 & 1.761 & 3.741 & 3.385 \\
\hline & 2 & 0.864 & 1.934 & 3.278 & 3.478 \\
\hline \multirow[t]{2}{*}{ BSE } & 0 & 0.717 & 1.180 & 2.985 & 2.755 \\
\hline & 2 & 0.427 & 1.435 & 2.670 & 2.905 \\
\hline \multirow[t]{2}{*}{$E_{b}$} & 0 & 0.574 & 0.581 & 0.756 & 0.630 \\
\hline & 2 & 0.437 & 0.499 & 0.608 & 0.573 \\
\hline
\end{tabular}

TABLE I: Calculated transition energies for the $(17,0)$ CNT using LDA, GW, and BSE. The exciton binding energy $E_{b}$ is calculated as the difference between the transition energies from GW and BSE.

Convergence with respect to k-point sampling is achieved with $128 \mathrm{k}$-points in the one-dimensional Brillouin zone. Also, the Coulomb potential is truncated [29, 30] in order to prevent tube-tube interactions or periodic image effects due to the use of a periodic supercell.

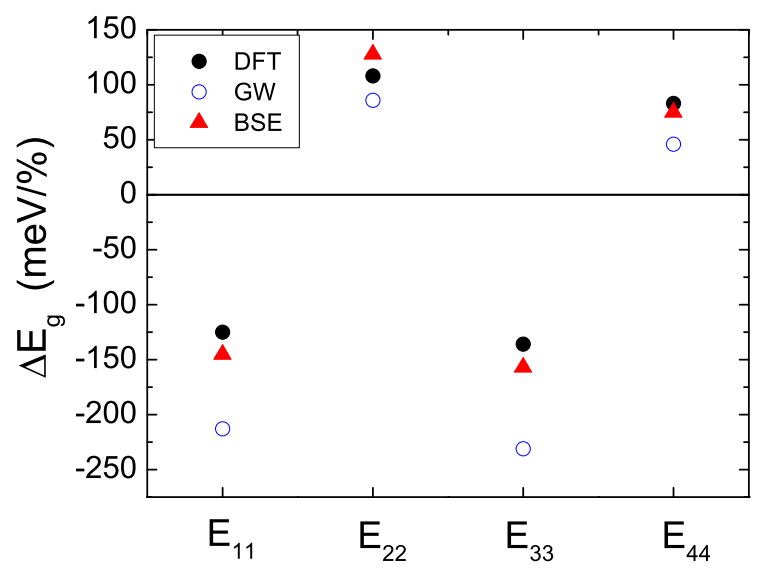

FIG. 2: Strain dependence of the DFT, GW, and BSE gaps for the $(17,0)$ CNT.

Figure 2 and Table 1 show the calculated GW gaps as a function of strain for the $(17,0) \mathrm{CNT}$. The qualitative dependence on strain is similar to that obtained within LDA, with the gaps increasing or decreasing with strain depending on the band index. However, we find that strain effects are much more complex within GW: for example $E_{11}$ and $E_{33}$ decrease by $213 \mathrm{meV} / \%$ and 231 $\mathrm{meV} / \%$ a much stronger change compared to LDA; on the other hand $E_{22}$ and $E_{44}$ increase by $86 \mathrm{meV} / \%$ and $46 \mathrm{meV} / \%$, much weaker than LDA predicts.

The above results for the $(17,0) \mathrm{CNT}$ are limited to two strain values due to the computational demands of the calculations. Tight-binding models predict a linear dependence of the bandgaps on strain for relatively small strains with changes in bandgaps independent of the tube diameter. To check whether this trend holds when many-body effects are included, we performed GW 


\begin{tabular}{lllll}
\hline \hline Strain (\%) & LDA & GW & BSE & $E_{b}$ \\
\hline 0 & 0.950 & 1.922 & 1.062 & 0.860 \\
2 & 0.694 & 1.540 & 0.775 & 0.764 \\
5 & 0.319 & 0.837 & 0.348 & 0.488 \\
\hline \hline
\end{tabular}

TABLE II: Energies (in eV) for the $E_{11}$ transition in the $(11,0)$ CNT calculated using LDA, GW, and BSE. The exciton binding energy $E_{b}$ is calculated as the difference between the GW and BSE energies.

calculations for three different values of the strain for the $E_{11}$ gap of the $(11,0)$ CNT. As shown in Fig. 3 and Table 2 , we obtain a linear dependence of the bandgap on strain in agreement with the tight-binding prediction for small strains and DFT. However, we find $\Delta E_{g}^{11}=-191$ $\mathrm{meV} / \%$ a value much larger than the LDA value of -127 $\mathrm{meV} / \%$; thus similar to the $(17,0) \mathrm{CNT}$, we find that many-body effects can significantly impact the electronic properties of the strained $(11,0)$ CNT.

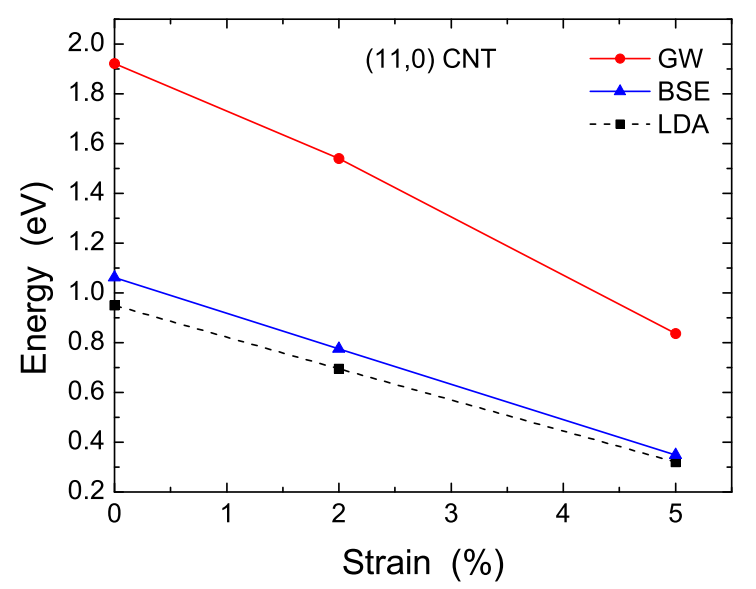

FIG. 3: Strain dependence of the $E_{11}$ transition for the $(11,0)$ CNT.

The differences between the GW and LDA results stem from the fact that with reduction (augmentation) of the fundamental bandgap there is an increase (decrease) in the dielectric screening $\varepsilon$ of the CNT. Consider the case where the fundamental bandgap decreases with strain: we plot in Fig. 4 the dielectric screening $\varepsilon^{-1}(q, \omega=0)$ for the $(11,0)$ CNT for strains of $0 \%, 2 \%$, and $5 \%$. The increased screening affects the screened Coulomb interaction $W=\varepsilon^{-1} v$ and hence the electron self-energy $\Sigma=i G W$ present in the many-body calculations. More exactly, the contribution $\Sigma_{g}$ of the electron self-energy to the quasiparticle bandgap $E_{g}^{G W}=E_{g}^{L D A}-V_{g}^{x c}+\Sigma_{g}$, decreases appreciably (same order of magnitude as the change in the LDA bandgap) upon strain: $\delta \Sigma_{g} \equiv \Sigma_{g}(\sigma)-$ $\Sigma_{g}(\sigma=0)<0$. This is a many-body effect not captured by the LDA exchange-correlation Kohn-Sham potential $V^{x c}$, and as expected we find $\delta V_{g}^{x c} / \delta \Sigma_{g} \ll 1$. Thus, the change in the fundamental quasiparticle bandgap upon strain as obtained within $\mathrm{GW}$ is more pronounced than the one obtained within a mean-field (LDA) theory, with appreciable contribution from self-energy corrections: $\delta E_{g}^{G W} \approx \delta E_{g}^{L D A}+\delta \Sigma_{g}$. For $E_{11}$ and $E_{33}$ this leads to a larger decrease in the bandgap compared to LDA since both $\delta E_{g}^{L D A}$ and $\delta \Sigma_{g}$ are negative; in contrast, $\delta E_{g}^{L D A}$ is positive for $E_{22}$ and $E_{44}$, and the still negative $\delta \Sigma_{g}$ leads to a smaller increase of the bandgap with strain.

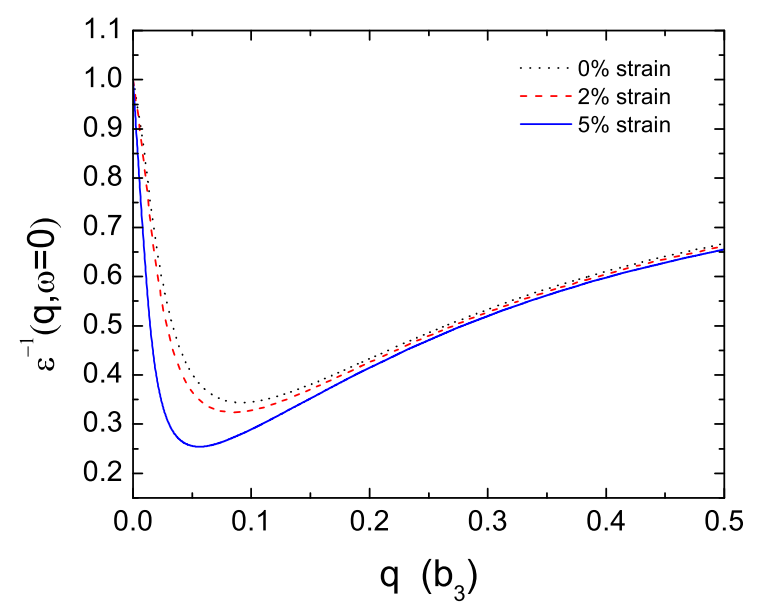

FIG. 4: Strain dependence of the dielectric screening $\varepsilon^{-1}$ for the $(11,0)$ CNT. $b_{3}$ is the length of the unit cell.

\section{IMPACT OF MANY-BODY EFFECTS ON OPTICAL PROPERTIES}

We next turn to the optical properties. To calculate these, we start from the GW results and couple them with the BSE. Both the BSE and GW calculations were performed using the BerkeleyGW package [31]. We solve the BSE for excitons within the static approximation for the dielectric screening and within the Tamm-Dancoff approximation for excitons [32]. Having obtained the excitonic properties one can then obtain the optical response of CNTs using the standard approach 2, 31]. The optical bandgap is equal to the quasiparticle bandgap minus the binding energy of the lowest bright exciton, a quantity which results from the overall attractive electron-hole interaction between the (quasi)electron and the (quasi)hole forming the exciton.

Figure 5 shows the optical absorbance for the $(17,0)$ CNT calculated within GW (no excitonic effects) and calculated within BSE (i.e. including excitonic effects) for the two lowest optical transitions. Here the absorbance is obtained from $A(\omega) \sim \omega \varepsilon_{2}(\omega)$ where $\varepsilon_{2}(\omega)$ is the imaginary part of $\varepsilon$. The peaks in the figure for the BSE results indicate the lowest energy bright exciton for light polarization parallel to the nanotube axis. At zero strain (Fig. 5a), the $E_{11}$ and $E_{22}$ transitions show strong manybody effects, with exciton binding energies of $574 \mathrm{meV}$ 
and $581 \mathrm{meV}$. Similar results are obtained for the $E_{33}$ and $E_{44}$ transitions (Table 1 ) with $E_{b}^{33}=756 \mathrm{meV}$ and $E_{b}^{33}=630 \mathrm{meV}$.

Upon application of strain (Fig. 5b) one can see that the $E_{11}$ exciton energy $\Omega_{11}$ decreases by $145 \mathrm{meV} / \%$ while $\Omega_{22}$ increases by $128 \mathrm{meV} / \%$. Results for $\Omega_{33}$ and $\Omega_{44}$ (Table 1) give values of $-157 \mathrm{meV} / \%$ and $+75 \mathrm{meV} / \%$, respectively. Thus, the qualitative trends observed from the GW calculations are maintained with the optical properties; however, because the quasiparticle bandgap and the exciton energy have a different dependence on strain, $d E_{g}^{G W} / d \sigma \neq d \Omega / d \sigma$, one can deduce that the exciton binding energy $E_{b}=E_{g}^{G W}-\Omega$ depends on strain. This can be seen in Fig. 5 and in Table 1 where all of the exciton binding energies are decreased under strain by amounts ranging from $28 \mathrm{meV} / \%$ for the $E_{44}$ transition to $74 \mathrm{meV} / \%$ for the $E_{33}$ transition. Much like the changes in the quasiparticle gap, the decrease in binding energy also stems from the change in dielectric screening upon applied strain. Indeed, the attractive interaction between the electron and hole forming the exciton is mediated by the screened Coulomb interaction $W=\varepsilon^{-1} v$, and because $\varepsilon$ is always determined by the lowest energy electronic bandgap, all of the optical transitions will be affected in the same way leading to the common decrease in binding energy.

It should also be noted that the exciton oscillator strength shows very small variation with strain. Since the oscillator strength is $\sim \Omega \mu_{a}^{2} / a$, with $\mu_{a}^{2} / a$ the squared exciton transition dipole matrix element per unit tube length 34], the implication is that $\mu_{a}^{2} / a$ strongly increases with increasing strain. Indeed, for the $(17,0)$ CNT, we find that $\mu_{a}^{2} / a$ increases from $\sim 3.9$ a.u. at zero strain to $\sim 6.8$ a.u. at $2 \%$ strain.

The optical results for the $(17,0)$ CNT can be generalized to the $(11,0) \mathrm{CNT}$ as well, at least for the lowest optical transition (Fig. 3 and Table 2). Indeed we find $d \Omega^{11} / d \sigma=-142 \mathrm{meV} / \%$ and a reduction of the exciton binding energy by severals tens of meV/\%. (At $2 \%$ the reduction in $E_{b}$ is about 3.5 times as large as that obtained using a tight-binding approach for excitons 19] for the $(11,0)$ CNT.) Furthermore, the exciton energy is found to depend linearly on strain, and turns out to be relatively close to the DFT result. As we discussed above, with decreasing bandgap the dielectric screening gets enhanced, and thus the binding between electron and hole decreases. This effect also explains why the change in optical gap $\Omega$ upon applied strain is similar to that obtained at the LDA level: it is due to cancellation effects between quasiparticle self-energy corrections and excitonic effects.

\section{SUMMARY}

In summary, we performed many-body ab initio calculations of the electronic and optical properties of semiconducting zigzag CNTs under uniaxial strain. We find that the fundamental electronic bandgap depends more
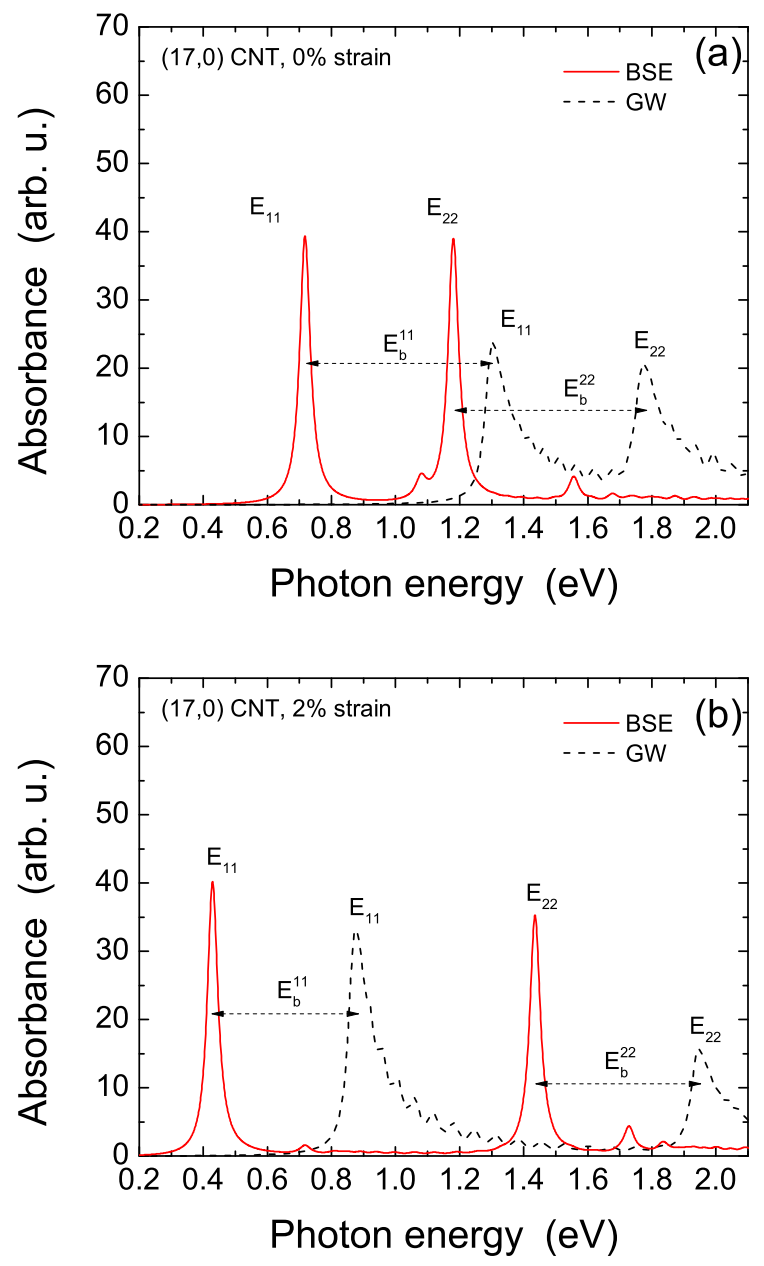

FIG. 5: Optical absorption spectrum of the (17,0) CNT calculated without the electron-hole interaction (GW) and with the electron-hole interaction (BSE). Panel (a) is for the unstrained case and panel (b) is for $2 \%$ strain.

strongly on strain than previously predicted by noninteracting models. In addition, we find that self-energy corrections generally decrease the bandgaps, which enhances or reduces the impact of strain compared to DFT depending on which transition is considered. Furthermore, the optical transitions are also found to be affected by many-body effects. In particular, the exciton binding energy decreases with increasing strain regardless of the transition, with variations of several tens of meVs per percent strain. More generally, our results indicate that quasiparticle and excitonic effects are strongly tied, and that the interpretation of optomechanical experiments in CNTs requires a more in-depth consideration of many-body effects. This is further supported by other many-body calculations on strained bulk [35, 36] and twodimensional materials [37, 38] where material-specific and dimensionality phenomena have been observed.

\section{ACKNOWLEDGEMENTS}

Work supported by the Laboratory Directed Research 
and Development program at Sandia National Laboratories, a multiprogram laboratory managed and operated by Sandia Corporation, a wholly owned subsidiary of Lockheed Martin Corporation, for the United States Department of Energy's National Nuclear Security Administration under Contract DE-AC04-94AL85000.
[1] F. Léonard, The Physics of Carbon Nanotube Devices, (William-Andrew, Norwich, 2009).

[2] C. D. Spataru, S. Ismail-Beigi, L. X. Benedict, and S. G. Louie, Phys. Rev. Lett. 92, 077402 (2004).

[3] T. Ando, J. Phys. Soc. Jpn. 66, 1066 (1997).

[4] E. Chang, G. Bussi, A. Ruini, E. Molinari, Phys. Rev. Lett. 92196401 (2004).

[5] V. Perebeinos, J. Tersoff, and Ph. Avouris, Phys. Rev. Lett. 92, 257402 (2004).

[6] H. Zhao, S. Mazumdar, Phys. Rev. Lett. 93, 157802 (2004).

[7] F. Wang, G. Dukovic, L. E. Brus, and T. F. Heinz, Science 308, 838 (2005).

[8] T. Ando, J. Phys. Soc. Jpn. 79, 024706 (2010).

[9] C. D. Spataru and F. Léonard, Phys. Rev. Lett. 104, $177402(2010)$.

[10] C. D. Spataru and F. Léonard, Chem. Phys. 81, 413 (2013).

[11] M. Rohlfing, Phys. Rev. Lett. 108, 087402 (2012).

[12] R. Heyd, A. Charlier, E. McRae, Phys. Rev. B 55, 6820 (1997).

[13] T. M. Tombler et al., Nature 405, 769 (2000).

[14] F.-Z. Zheng, Z.-Y. Zhou, X. Yang, Y.-K. Tang, and Y. Wu, IEEE Trans. Nanotech. 10, 694 (2011).

[15] H. Maki, T. Sato, and K. Ishibashi, Nano Lett. 7, 890 (2007).

[16] M. Huang et al., Phys. Rev. Lett. 100, 136803 (2008).

[17] S. M. Kaniber, L. Song, J. P. Kotthaus, and A. W. Holleitner, Appl. Phys. Lett. 94, 261106 (2009).

[18] G. Yu, Y. Jia, J. Dong, J. Phys.: Condens. Matter 19, 266222 (2007).

[19] G.-L. Yu, Y.-L. Jia, Chin. Phys. Lett. 26, 037102 (2009).
[20] G. Yu, Y. Jia, G. Tang, Physica B 405, 167 (2010).

[21] T. Ando, J. Phys. Soc. Jpn 73, 3351 (2004).

[22] P. Giannozzi et al., J.Phys.:Condens.Matter 21, 395502 (2009).

[23] R. Saito, G. Dresselhaus, M. S. Dresselhaus, Phys. Rev. B 61, 2981 (2000).

[24] S. Sreekala, X.-H. Peng, P. M. Ajayan, and S. K. Nayak, Phys. Rev. B 77, 155434 (2008).

[25] P. K. Valavala, D. Banyai, M. Seel, and R. Pati, Phys. Rev. B 78, 235430 (2008).

[26] L. Yang and J. Han, Phys. Rev. Lett. 85, 154 (2000).

[27] M.S. Hybersten, S.G. Louie, Phys. Rev. B 34, 5390 (1986).

[28] J. Deslippe et al.,Phys. Rev. B 87, 165124 (2013).

[29] S. Ismail-Beigi, Phys. Rev. B 73, 233103 (2006).

[30] C. D. Spataru et al., Appl. Phys. A 78, 1129 (2005).

[31] J. Deslippe et al., Comp. Phys. Comm. 183, 12692012 (2012).

[32] A. Fetter and J.D. Walecka, Quantum Theory of Many Particle Systems (McGraw-Hill, San Francisco, 1971).

[33] M. F. Lin, D. S. Chuu, C. S. Huang, Y. K. Lin, and K. W.-K. Shung, Phys. Rev. B 53, 15493 (1996).

[34] C. D. Spataru, S. Ismail-Beigi, R. B. Capaz, and S. G. Louie, Phys. Rev. Lett. 95, 247402 (2005).

[35] L. Thulin and J. Guerra, Phys. Rev. B 77, 195112 (2008).

[36] T. Cheiwchanchamnangij and W. R. L. Lambrecht, Phys. Rev. B 84, 035203 (2011).

[37] H. Shi, H. Pan,Y.-W. Zhang, and B. I. Yakobson, Phys. Rev. B 87, 155304 (2013).

[38] Y. Liang, S. Huang, and L. Yang, J. Mater. Res. 27, 403 (2012). 